\title{
Contents
}

\section{Part I Introduction to Kinetics}

and Many-Body Theory

$1 \quad$ Boltzmann Equation $\ldots \ldots \ldots \ldots \ldots \ldots \ldots \ldots \ldots \ldots \ldots \ldots$

1.1 Heuristic Derivation

of the Semiclassical Boltzmann Equation ............. 3

1.2 Approach to Equilibrium: H-Theorem .............. 5

1.3 Linearization: Eigenfunction Expansion............. 8

2 Numerical Solutions of the Boltzmann Equation ......... 11

$2.1 \quad$ Introduction . . . . . . . . . . . . . . . . . . . . . . . . 11

2.2 Linearized Coulomb Boltzmann Kinetics

of a $2 \mathrm{D}$ Electron Gas......................... 12

$2.3 \quad$ Ensemble Monte Carlo Simulation ............... 20

2.3.1 General Theory ....................... 20

2.3.2 Simulation of the Relaxation Kinetics of a 2D Electron Gas...................... 23

$2.4 N^{+} N^{-} N^{+}$Structure: Boltzmann Equation Analysis . . . . 29

$3 \quad$ Equilibrium Green Function Theory . . . . . . . . . . . . . 35

$3.1 \quad$ Second Quantization ........................ 35

3.2 Density Matrix Equations:

An Elementary Derivation

of a Non-Markovian Quantum Kinetic Equation ......... 38

$3.3 \quad$ Green Functions ......................... 41

3.3.1 Examples of Measurable Quantities ........... 43

$3.4 \quad$ Fluctuation-Dissipation Theorem ................. 45

3.5 Perturbation Expansion of the Green Function ......... 47

3.6 Examples of Simple Solvable Models .............. 50

3.6.1 Free-Particle Green Function ................. 50

3.6.2 Resonant-Level Model .......................... 50 
XVI Contents

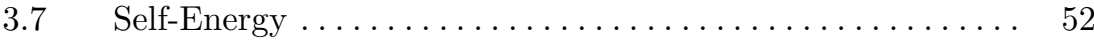

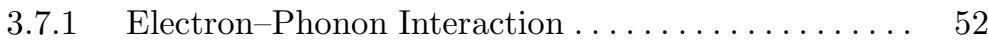

3.7.2 Elastic Impurity System: Impurity Averaging . . . . . 54

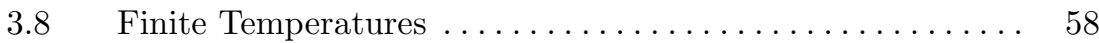

Part II Nonequilibrium Many-Body Theory

4 Contour-Ordered Green Functions ................... 63

$4.1 \quad$ General Remarks ...................... 63

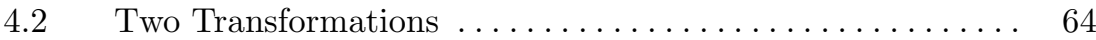

$4.3 \quad$ Analytic Continuation.................... 69

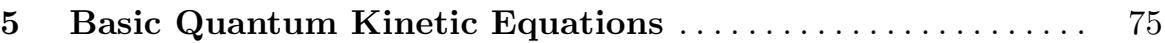

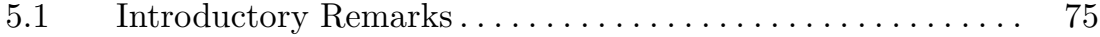

5.2 The Kadanoff-Baym Formulation . . . . . . . . . . . . . . 75

$5.3 \quad$ Keldysh Formulation . . . . . . . . . . . . . . . . . 77

6 Boltzmann Limit $\ldots \ldots \ldots \ldots \ldots \ldots \ldots \ldots \ldots \ldots \ldots \ldots$

$6.1 \quad$ Gradient Expansion . . . . . . . . . . . . . . . . . . 79

6.2 Quasiparticle Approximation .................... 81

6.3 Recovery of the Boltzmann Equation ............. 82

$7 \quad$ Gauge Invariance $\ldots \ldots \ldots \ldots \ldots \ldots \ldots \ldots \ldots \ldots \ldots$

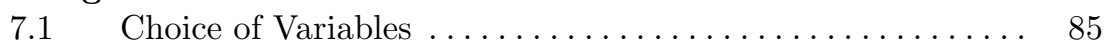

7.2 Gauge Invariant Quantum Kinetic Equation ... . . . . . . . 87

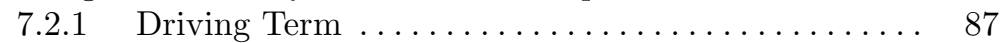

$7.2 .2 \quad$ Collision Term ................... 90

$7.3 \quad$ Retarded Green Function . . . . . . . . . . . . . . . . . . . . . . . 91

8 Quantum Distribution Functions................. 93

8.1 Relation to Observables, and the Wigner Function ....... . 93

8.2 Generalized Kadanoff-Baym Ansatz ... . . . . . . . . . . . . . 94

8.3 Summary of the Main Formal Results . . . . . . . . . . . . 97

Part III Quantum Transport

in Semiconductors

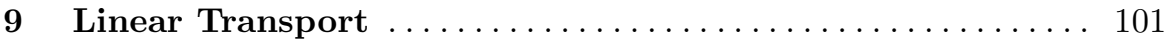

9.1 Quantum Boltzmann Equation .................. 101

9.2 Linear Conductivity

of Electron-Elastic Impurity Systems . . . . . . . . . . . . . . . . . . 104

9.2.1 Kubo Formula ... . . . . . . . . . . . . . . . . 105

9.2.2 Quantum Kinetic Formulation.... . . . . . . . . . 109

9.3 Weak Localization Corrections to Electrical Conductivity . . 111 
10 Field-Dependent Green Functions . . . . . . . . . . . . . . 115

10.1 Free Green Functions

and Spectral Functions in an Electric Field ............ 115

10.2 A Model for Dynamical Disorder:

The Gaussian White Noise Model . . . . . . . . . . . . . 121

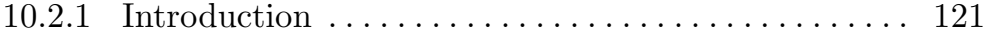

10.2.2 Determination of the Retarded Green Function . . . 121

10.2.3 Kinetic Equation for the GWN . . . . . . . . . . . . 123

10.2.4 Other Transport Properties . . . . . . . . . . . . 127

10.3 Introduction to High-Field Transport in Semiconductors . . 129

10.4 Resonant-Level Model in High Electric Fields . . . . . . . . . 131

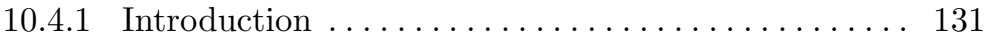

10.4.2 Retarded Green Function:

Single Impurity Problem . . . . . . . . . . . . . . . . . 131

10.4.3 Retarded Green Function:

Dilute Concentration of Impurities . . . . . . . . . . . 133

10.4.4 Analytic Continuation................... 140

10.4.5 Quantum Kinetic Equation .................. 141

10.5 Quantum Kinetic Equation

for Electron-Phonon Systems . . . . . . . . . . . . . . . 144

10.6 An Application:

Collision Broadening for a Model Semiconductor ........ 148

10.6.1 Analytical Considerations . . . . . . . . . . . . . . . 148

10.6.2 A Simple Model:

Optical Phonon Emission at $T=0 \ldots \ldots \ldots \ldots \ldots$

10.7 Spatially Inhomogeneous Systems . . . . . . . . . . . . . . 151

11 Optical Absorption in Intense THz Fields . . . . . . . . . . . . . . . 157

11.1 Introductory Remarks . . . . . . . . . . . . . . . . . . 157

11.2 Optical Absorption as a Response Function . . . . . . . . . . . 158

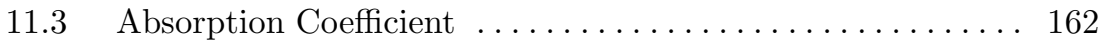

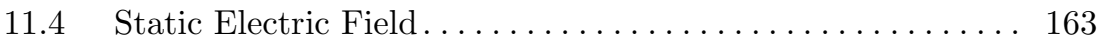

11.5 Harmonically Varying External Electric Fields . . . . . . . . . 164

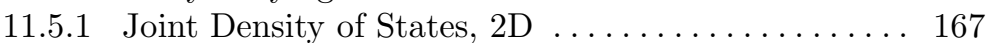

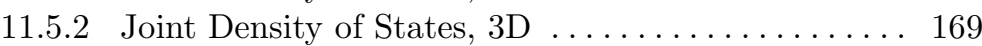

11.6 Dynamical Franz-Keldysh Effect: Excitonic Effects . . . . . 171

11.6.1 Matrix Truncation ..................... 172

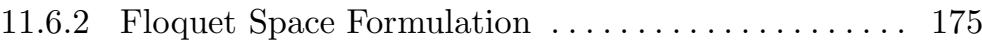

12 Transport in Mesoscopic Semiconductor Structures . . . . . . 181

12.1 Introduction $\ldots \ldots \ldots \ldots \ldots \ldots \ldots \ldots \ldots \ldots \ldots \ldots \ldots$

12.2 Nonequilibrium Techniques

in Mesoscopic Tunneling Structures . . . . . . . . . . . . . . . . . . 184

12.3 Model Hamiltonian . . . . . . . . . . . . . . . . . . . . . . . . . . . . . . 185

12.4 General Expression for the Current . . . . . . . . . . . 186 
XVIII Contents

12.5 Current Conservation . . . . . . . . . . . . . . . . . . . 191

12.6 Noninteracting Resonant-Level Model . . . . . . . . . . . . . . . . . . 192

12.7 Density Functional Theory

and Modeling of Molecular Electronics . . . . . . . . . . . . 195

12.8 Resonant Tunneling

with Electron-Phonon Interactions . . . . . . . . . . 196

12.9 Transport in a Semiconductor Superlattice . . . . . . . . . . . 198

12.10 Transport in Atomic Gold Wires:

Signature of Coupling to Vibrational Modes. . . . . . . . . . 202

12.11 Transport Through a Coulomb Island . . . . . . . . . . . . . . 205

13 Time-Dependent Phenomena . . . . . . . . . . . . . . . . . 213

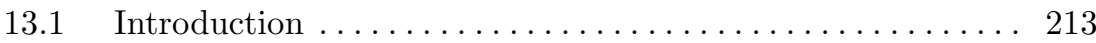

13.2 Applicability to Experiments . . . . . . . . . . . . . 214

13.3 Mathematical Formulation ..................... 215

13.4 Average Current........................ 217

13.5 Time-Dependent Resonant-Level Model . . . . . . . . . . . . . . . 218

13.5.1 Response to Harmonic Modulation . . . . . . . . . . . . 221

13.5.2 Response to Step-Like Modulation . . . . . . . . . . . . . 224

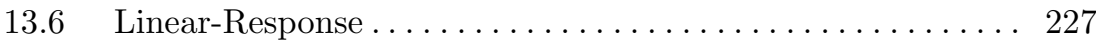

$13.7 \quad$ Fluctuating Energy Levels . . . . . . . . . . . . . . . . . . . . 229

13.8 Noise . . . . . . . . . . . . . . . . . . . . . . 230

13.8.1 The Disconnected Terms ............... 235

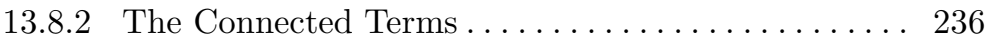

Part IV Theory of Ultrafast Kinetics

in Laser-Excited Semiconductors

14 Optical Free-Carrier Interband Kinetics

in Semiconductors . . . . . . . . . . . . . . . . . . . . . . 243

14.1 Interband Transitions in Direct-Gap Semiconductors . . . . . 243

14.1.1 Reduced Density Matrices ................ 243

14.1.2 Nonequilibrium Green Functions.............. 245

14.1.3 Calculations of the Two-Time-Dependent

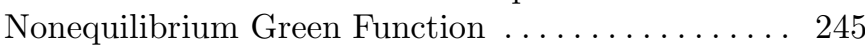

14.1.4 Replacement of Two-Time Propagators

by a One-Time Density Matrix

and a Two-Time Spectral Function .......... 246

14.2 Free-Carrier Kinetics Under Laser-Pulse Excitation . . . . . . 251

14.3 The Optical Free-Carrier Bloch Equations . . . . . . . . . . . 255

15 Interband Quantum Kinetics with LO-Phonon Scattering . 259

15.1 Derivation of the Interband Quantum Kinetic Equations . . 259

15.2 The Spectral Green Functions $G_{\mu \nu}^{\mathrm{r}}$ and $G_{\mu \nu}^{\mathrm{a}} \ldots \ldots \ldots 266$ 
15.2.1 Free-Particle Retarded Green Function ......... 266

15.2.2 Retarded GF in the Mean-Field Approximation . . . 267

15.2.3 Spectra of Retarded Gfs . . . . . . . . . . . . . . 269

15.2.4 Dephasing of Retarded Green Functions ... . . . . . . 274

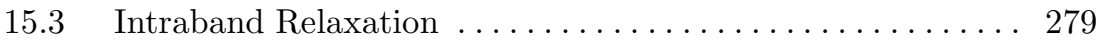

15.4 Interband-Polarization Dephasing. . . . . . . . . . . 281

$15.5 \quad$ Numerical Strategies . . . . . . . . . . . . . . . . . . 283

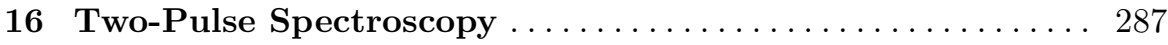

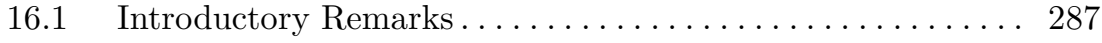

16.2 Thin Samples . . . . . . . . . . . . . . . . . . . . . . . . . . . . 289

16.3 Low-Intensity Two-Beam Experiments . . . . . . . . . . . . . . 290

16.3.1 LO-Phonon Relaxation Cascades .............. 291

16.3.2 LO-Phonon Quantum Beats in FWM . . . . . . . 292

16.3.3 Two-Time Electron-Phonon Quantum Kinetics ... . 294

17 Coulomb Quantum Kinetics

in a Dense Electron-Hole Plasma . . . . . . . . . . . . . . . . . 301

17.1 Introduction $\ldots \ldots \ldots \ldots \ldots \ldots \ldots \ldots \ldots \ldots \ldots \ldots \ldots \ldots \ldots \ldots$

17.2 Screening in the Nonequilibrium GF Theory . . . . . . . . . 302

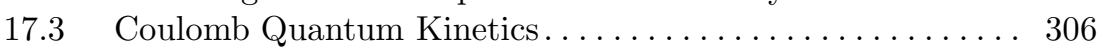

17.4 Plasmon-Pole Approximation

for the Two-Time-Dependent Potential . . . . . . . . . . . . 309

17.4.1 Parametric Plasma Oscillations . . . . . . . . . . 311

17.4.2 Instantaneous Static Potential Approximation .... . 313

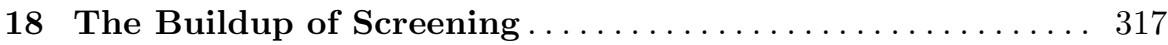

18.1 Screening of the Coulomb Interaction . . . . . . . . . 317

18.1.1 Calculations of the Two-Time-Dependent

Screened Potential ..................... 318

18.1.2 Femtosecond Optical Pump and $\mathrm{THz}$ Probe Spectroscopy ............. 320

18.2 Time-Dependent Screening of Phonon-Mediated

and Coulomb Interactions $\ldots \ldots \ldots \ldots \ldots \ldots \ldots \ldots$

18.2.1 Buildup of the Phonon-Plasmon Mixed Modes ... . 324

19 Femtosecond Four-Wave Mixing with Dense Plasmas . . . . 331

19.1 Time-Resolved Four-Wave Mixing . . . . . . . . . . . . 331

19.2 Time-Integrated Four-Wave Mixing . . . . . . . . . . . . . . . . . 334

19.3 Four-Wave Mixing with Coherent Control . . . . . . . . . . . 335

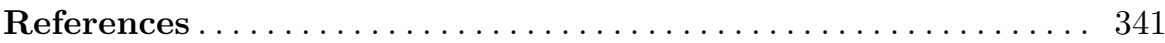

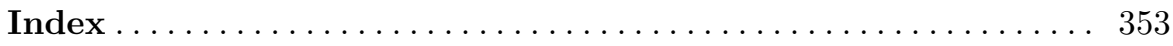

